\title{
An integrated device for fast and sensitive immunosuppressant detection
}

\author{
Sara Tombelli ${ }^{1}$ - Cosimo Trono ${ }^{1}$ (D) Simone Berneschi ${ }^{1} \cdot$ Chiara Berrettoni $^{1} \cdot$ Ambra Giannetti $^{1} \cdot$ Romeo Bernini $^{2}$. \\ Gianluca Persichetti ${ }^{2} \cdot$ Genni Testa $^{2} \cdot$ Guillermo Orellana $^{3}$. Francesca Salis ${ }^{3}$. Susanne Weber ${ }^{4} \cdot$ Peter B. Luppa $^{4}$. \\ Giampiero Porro ${ }^{5}$. Giovanna Quarto ${ }^{5}$. Markus Schubert ${ }^{6} \cdot$ Marcel Berner $^{7} \cdot$ Paulo P. Freitas $^{8}$. Susana Cardoso ${ }^{8}$. \\ Fernando Franco $^{8}$. Vânia Silverio ${ }^{8} \cdot$ Maria Lopez-Martinez $^{8}$. Urs Hilbig ${ }^{9} \cdot$ Kathrin Freudenberger $^{9}$. \\ Günter Gauglitz ${ }^{9} \cdot$ Holger Becker $^{10} \cdot$ Claudia Gärtner $^{10} \cdot$ Mark T. O'Connell $^{11} \cdot$ Francesco Baldini $^{1}$
}

Received: 23 September 2021 / Revised: 22 November 2021 / Accepted: 10 December 2021 / Published online: 22 December 2021

(c) The Author(s) 2021

\begin{abstract}
The present paper describes a compact point of care (POC) optical device for therapeutic drug monitoring (TDM). The core of the device is a disposable plastic chip where an immunoassay for the determination of immunosuppressants takes place. The chip is designed in order to have ten parallel microchannels allowing the simultaneous detection of more than one analyte with replicate measurements. The device is equipped with a microfluidic system, which provides sample mixing with the necessary chemicals and pumping samples, reagents and buffers into the measurement chip, and with integrated thin film amorphous silicon photodiodes for the fluorescence detection. Submicrometric fluorescent magnetic particles are used as support in the immunoassay in order to improve the efficiency of the assay. In particular, the magnetic feature is used to concentrate the antibody onto the sensing layer leading to a much faster implementation of the assay, while the fluorescent feature is used to increase the optical signal leading to a larger optical dynamic change and consequently a better sensitivity and a lower limit of detection. The design and development of the whole integrated optical device are here illustrated. In addition, detection of mycophenolic acid and cyclosporine A in spiked solutions and in microdialysate samples from patient blood with the implemented device are reported.
\end{abstract}

Keywords Immunosuppressant $\cdot \mathrm{POCT} \cdot$ Fluorescence $\cdot$ Cyclosporine A $\cdot$ Mycophenolic acid

Published in the topical collection Point-of-Care Testing with guest editors Oliver Hayden, Peter B. Luppa and Junhong Min.

Cosimo Trono

c.trono@ifac.cnr.it

1 Institute of Applied Physics "Nello Carrara", CNR-IFAC, Via Madonna del Piano 10, 50019 Sesto Fiorentino, Italy

2 Institute for Electromagnetic Sensing of the Environment, CNR-IREA, Via Diocleziano 328, 80124 Napoli, Italy

3 Department of Organic Chemistry, Faculty of Chemistry, Universidad Complutense de Madrid, 28040 Madrid, Spain

4 Institute of Clinical Chemistry and Pathobiochemistry, Klinikum rechts der Isar, Technische Universität München, Marchioninistrasse 15, 8000 Munich, Germany

5 Datamed Srl, Via Grandi 4/6, 20068 - Peschiera Borromeo, Milan, Italy

6 Institute for Photovoltaics and Research Center SCoPE, University of Stuttgart, 70569 Stuttgart, Germany
7 Innovative Pyrotechnik GmbH, Steinwerkstraße 2, 71139 Ehningen, Germany

8 Instituto de Engenharia de Sistemas e Computadores-Microsistemas e Nanotecnologias, R.Alves Redol 9, 1000-027 Lisbon, Portugal

9 Institute for Physical and Theoretical Chemistry, Eberhard Karls University, Auf der Morgenstelle 18, 72076 Tübingen, Germany

10 microfluidic ChipShop GmbH, Stockholmer Str. 20, 07747 Jena, Germany

11 Cornel Medical Limited, 17 Church Walk, St Neots, Cambridgeshire PE19 1JH, UK 


\section{Introduction}

Point of care testing (POCT) devices measure analytes of clinical interest close to the patient [1-3], providing physicians the possibility to achieve the correct diagnosis or to administer the right therapy in a relatively short time and their use is more and more diffusing with the approval of the competent authorities responsible for overseeing the enforcement of medical device regulations. They are generally able to perform single-shot measurements on different biological matrices (blood, urine, saliva, etc.) while a frequent/near-continuous measurement remains a challenging task in the clinical practice, so far confined to laboratory and at prototype level, with the exclusion of rare exceptions such as the detection of glucose in diabetic patients. The advent of personalised medicine has shown the importance, if not the compelling need, to tailor the right therapeutic strategy for the right person at the right time, and therapeutic drug monitoring (TDM) with POCT devices is becoming more and more important in many pathologies, very often associated to the need of frequent/ near-continuous measurement of the analyte of interest [4-6].

Immunosuppressant monitoring after organ transplantation is a mandatory issue for the life of transplanted patients: if levels of immunosuppressive drugs are too high, the inhibition of the immune system is excessive and the patient may be unable to fight off infections, while if the dosage is too low there is a danger of organ rejection. Drugs of interest are chemicals with a narrow therapeutic window, which regularly pose considerable problems in initial and ongoing dosing $[7,8]$. These drugs are often subject to polymorphic metabolism with considerable inter- and intra-individual variability requiring TDM for personalised dosage [9]. As these drugs are highly sequestered in erythrocytes and leukocytes, and also reversibly bound to plasma proteins, their concentration is routinely measured nowadays in haemolysed whole blood samples, by means of laboratory techniques, such as liquid chromatography-tandem mass spectrometry (LC-MS/MS) and fluorescence or chemiluminescence immunoassays $[8,10$, 11]. This implies that TDM is usually performed with only one blood sample per dosing interval taken either prior or $1-2 \mathrm{~h}$ after drug administration. The drug concentration is subsequently evaluated in the laboratory and barely available just before the next dose. In the best case, this approach provides information on the trough and peak levels, which are the lowest and highest concentration reached by a drug before the next dose is administered, respectively. Recent studies have shown that more accurate clinical indication is given by the area under the curve (AUC) of the drug concentration vs time, the determination of which would need a frequent - ideally continuous - monitoring, as this value is better correlated with both the efficiency and side-effects of the administered drug therapy than the trough level [12-14].

On this basis, it is clear that a device capable to measure frequently immunosuppressants close to transplanted patients is a strong plea of physicians in transplantation surgery for the determination of the right dosage of the administered drugs in the first period after transplantation, increasing the number of relevant or evolving clinical parameters to be analysed with POCT devices [15]. Among the typical immunosuppressants, the calcineurin inhibitors cyclosporine A [16] and tacrolimus (FK 506) $[17,18]$ are of particular interest, as well as mycophenolic acid [19, 20], which inhibits an enzyme needed for the growth of T- and B-cells. The device must be also able to perform simultaneous measurement of more than one analyte, since the administration of a mixture of immunosuppressants instead of a single drug is often the followed protocol in the post-operative treatment of transplanted patients [21, 22]. It is also important to observe that the free fraction of immunosuppressants circulating in blood has been recognised the responsible for their pharmacological activity, as well as for associated side effects [23]. The reason why the free fraction is not addressed by TDM not only lies in the lack of relevant reference values available [24] but also because of its very low concentration, much lower (just a few \%) than the total concentration in the blood, i.e. down to $\mathrm{ng} / \mathrm{mL}$ or less. In a recent paper [25], intravascular microdialysis has been demonstrated a reliable technique to extract the free fraction of immunosuppressants paving the way towards quasi-continuous therapeutic drug monitoring in transplanted patient; this can provide a more effective control of the drug uptake by the transplanted patients in a standard routine, unthinkable to be performed nowadays, and quickly allowing the correct dosing of the administered drugs, which is essential for the therapy outcome and the patient's safety.

The need for quasi-continuous/frequent measurements makes it necessary to adopt suitable strategies to reduce as much as possible the assay time. Magnetic particles have been shown to be an efficient tool to accelerate the assay time while maintaining high sensitivity, providing the possibility to reduce the incubation time and preserving high binding efficiencies $[26,27]$. Furthermore, the addition of a fluorescent label makes their easy integration in optical bioassays possible [28], which is an important aspect to be considered since optical biosensors are playing a relevant role in TDM $[29,30]$.

Having in mind to fill the gap nowadays present in TDMPOCT devices, the present paper describes a compact optical device capable to perform a fluorescence-based immunoassay, using a disposable plastic chip where the assay takes place for the determination of immunosuppressants. The 
particular structure of the chip with ten parallel microchannels allows the simultaneous detection of more than one analyte with replicate measurements. The device is equipped with microfluidic circuitry, which handles the sample mixing with the necessary chemicals using a second suitable chip and pumping it into the measurement chip, and with integrated thin film amorphous silicon photodiodes for the fluorescence detection. Submicrometric fluorescent magnetic particles are used as support in the immunoassay in order to improve the efficiency of the assay. In particular, the magnetic feature is used to concentrate the antibody onto the sensing layer leading to a much faster implementation of the assay, while the fluorescent feature is used to increase the optical signal leading to a larger optical dynamic change and consequently a better sensitivity and a lower limit of detection. For the purpose to perform quasi-continuous/frequent measurements over a period of several hours on the same patient with only one chip, the regeneration of the chip was also implemented. In this way, the developed chip could be considered as a 'single-patient' chip, close to the concept of single-use chip, which is usually required in clinical chemistry. The use of the device with microdialysate samples allowing continuous sampling for $12-48 \mathrm{~h}$ would ease the assessment of the AUC, which is considered increasingly important for pharmacokinetic monitoring of immunosuppressive drugs [14].

\section{Experimental}

\section{The optical device}

The device was composed of an optoelectronic system which comprised an excitation and detection module and a fluidic circuit for handling the sample and it was equipped with a permanent magnet moving system. The core of the device, where the fluorescence-based bioassay took place, was a novel plastic chip equipped with ten separate microfluidic channels which allowed the simultaneous measurement of different immunosuppressants. Each microfluidic channel was integrated with an illumination system via an optical fibre and then coupled with a photodiode through an optical filter. Finally, the bottom of the chip was connected with an array of ten permanent magnets (Fig. 1). Details on the design, optimisation and final integration of the device are given in the 'Results and discussion' section.

\section{Materials and protocols}

Fluorescent magnetic particles Commercial magnetic 300 $\mathrm{nm}$ diameter particles (Estapor ${ }^{\circledR}$, Merck Millipore) with a polystyrene core were used. The polystyrene particles were decorated with ferrite nanograins (ca. $8-10 \mathrm{~nm}$ ) that

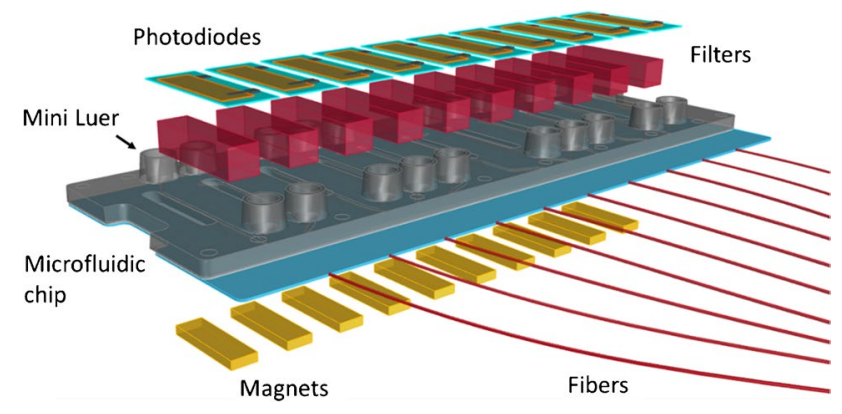

Fig. 1 Exploded view of the core of the POCT system

conferred superparamagnetic properties. They were also functionalised with carboxylic groups on the surface, which were needed for further bioconjugation. These non-fluorescent particles were doped with a hydrophobic boron-dipyrromethane BODIPY 641 dye by a swelling procedure [31], yielding brilliant fluorescent beads $\left(\lambda_{\mathrm{ex}}{ }^{\max }=640 \mathrm{~nm} ; \lambda_{\mathrm{em}}{ }^{\max }\right.$ $=680 \mathrm{~nm}$ ) despite the dense ferrite shell on the surface.

Immunosuppressants and their derivatives Mycophenolic acid (MPA), cyclosporine A (CsA) and tacrolimus powders used as standards were purchased from Abcam. The cyclosporine A carboxylic acid derivative $\left(\mathrm{CsA}-\mathrm{O}-\mathrm{CO}_{2} \mathrm{H}\right)$ was prepared as described in [25]. The following antibodies were used: polyclonal sheep anti-mycophenolic acid IgG antibody (Abcam) and monoclonal mouse anti-cyclosporine A IgG1 antibody (clone CSZ2.22) (Abcam).

Other reagents and solutions 1 -Ethyl-( $N, N$-dimethylamino) propylcarbodiimide hydrochloride (EDC) and $\mathrm{N}$-hydroxysuccinimide (NHS), Tween 20, reagents for buffer preparation and sodium dodecyl sulphate (SDS) solution were all from Sigma and Lipofundin MCT/LCT 20\% from B. Braun Melsungen.

Immobilisation of antibodies onto the magnetic fluorescent particles For the immobilisation of antibodies onto the fluorescent magnetic particles (FMPs), the carboxylic groups on the FMPs were activated by means of EDC $(40 \mathrm{mg} / \mathrm{mL}$ in MES buffer) for $10 \mathrm{~min}$. After separation with an external permanent magnet the activated FMPs were incubated with the immunosuppressant-specific antibody $(10 \mu \mathrm{g} / \mathrm{mL})$ for $2 \mathrm{~h}$. After separation, the FMPs were washed 3 times with phosphate-buffered saline (PBS), blocked with Pierce protein-free blocking buffer (ThermoFisher Scientific, Monza, Italy) and washed again with PBS. After washing, the coated FMPs were stored at $4^{\circ} \mathrm{C}$.

Immobilisation of immunosuppressants onto the microfluidic channels of the chip The channels of the chip were modified with MPA or the CsA-O- $\mathrm{CO}_{2} \mathrm{H}$ derivative via 
carbodiimide crosslinking chemistry [25]. The carboxylic groups of the antigen were firstly activated by a freshly prepared aqueous solution containing $200 \mathrm{mM}$ EDC and $50 \mathrm{mM}$ NHS and then flowed through the channels for $1 \mathrm{~h}$ using a peristaltic pump (Gilson, Milan, Italy). After immobilisation, the channels were washed with PBS and blocked with undiluted protein-free blocking buffer to prevent any potential non-specific adsorption.

Assay general protocol The general protocol of the assay for the determination of the immunosuppressants inside the integrated optical device is detailed in Fig. 2. The perfusate/microdialysate sample containing the immunosuppressants was mixed and incubated with the FMPs carrying the immunosuppressant-specific antibodies outside the measuring chip, with the use of a proper mixing chip; the mixed sample/FMPs was then pumped sequentially into the different microfluidic channels of the chip. During this step, the magnets at the bottom of the channels were moved close to the chip and the antibodies onto the particles that were not bound to the immunosuppressant, interacted with the immunosuppressant immobilised onto the surface of the microchannel. The magnets were then moved away from the chip and washing with buffer was conducted in each microfluidic channel. During this step, the antibodies, and consequently the FMPs, which did not interact with the analyte onto the surface of the channels were washed away. Finally, the fluorescence measurement was performed, simultaneously onto all the microfluidic channels.

After the measurement, regeneration of the channels surface was carried out, followed by washing with buffer and pumping of air inside all the fluidics in order to assure the same starting conditions for each measurement (each sample).

Details on the fluidic procedures used for the final assays conducted inside the integrated optical device are described in the following section.
Fig. 2 Scheme of the different steps of the on-chip immunoassay

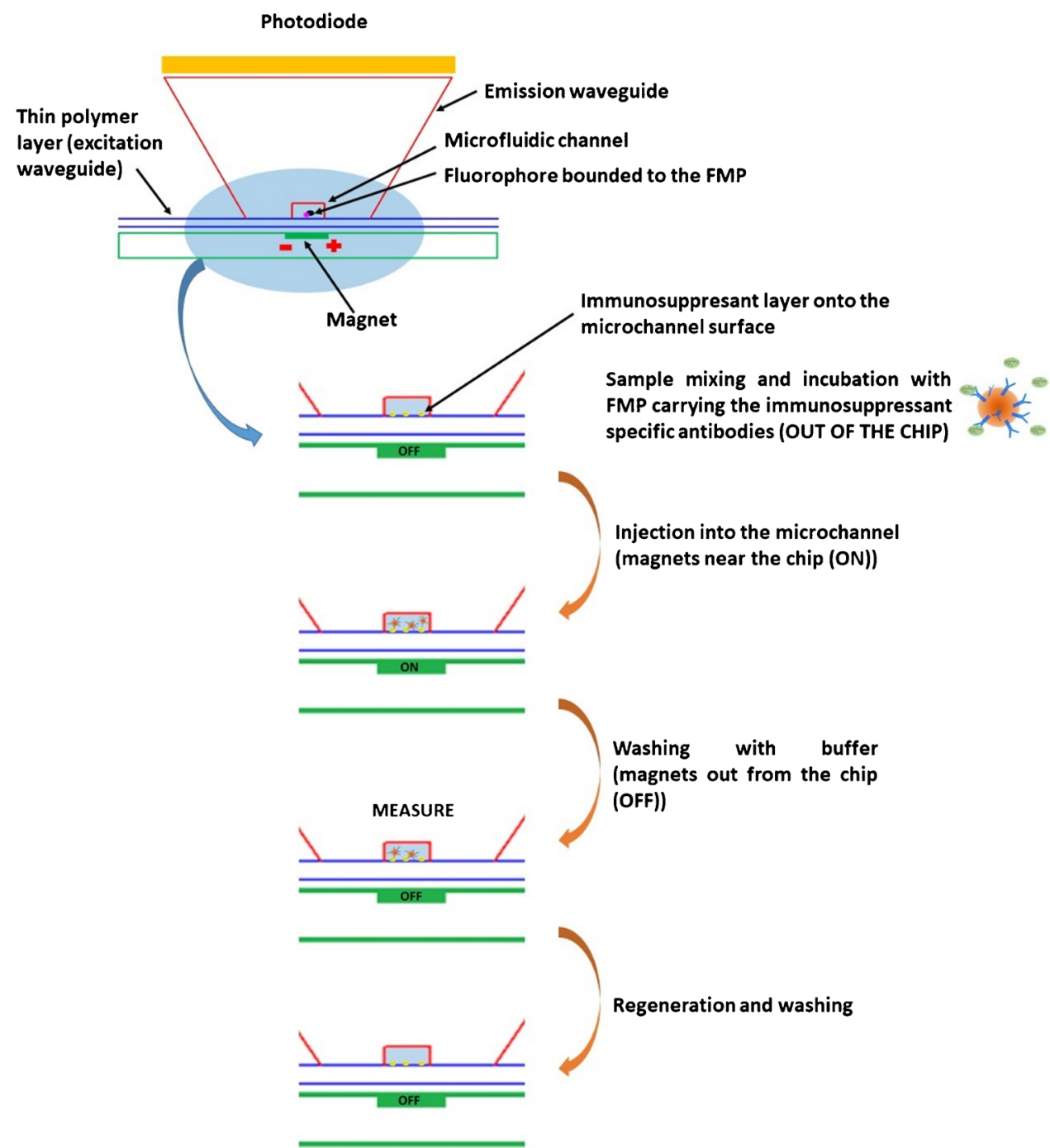


Spiked samples and real microdialysate samples CsA and MPA were spiked into 20\% Lipofundin MCT/LCT (20\%) in isotonic saline solution at different concentrations (range: 0-30 ng/mL for MPA and 0-10 ng/mL for CsA). Real microdialysate samples were collected from ex vivo microdialysis of whole blood samples of immunosuppressed patients by using a MicroEye ${ }^{\circledR}$ catheter $(9,000$ Da cut-off; Probe Scientific, Bedford, UK) combined with a Technic I syringe pump (AMV technics, Předklášteří, Czech Republic) set to a flow rate of $1.6 \mu \mathrm{L} / \mathrm{min}$ (see [25] for more details) and using $20 \%$ Lipofundin MCT/LCT (20\%) in isotonic saline solution as perfusate.

Fluidic protocol for the sample analysis in the integrated device The microfluidic chip, functionalised with $\mathrm{NH}_{2}$ groups, was coated with MPA in four channels and with CsA-O- $\mathrm{CO}_{2} \mathrm{H}$ in other four by using a peristaltic pump before its loading into the instrument. The CsA- and MPAcoated chip was then loaded into the optical device for its connection to the fluidic system. The modified surface of the channels was subsequently exposed to solutions containing the anti-CsA and anti-MPA antibody-coated FMPs mixed and incubated with Lipofundin (20\% in PBS), with the real samples (final dilution 1:1) or with different concentrations of CsA and MPA diluted in 20\% Lipofundin.

The following fluidic procedure was used for the binding phase of the assay:

1. Filling of the fluidic line from the solution vial to the fluidic switch used for the channel selection (60 s);

2. Sequential filling of the channels ( $10 \mathrm{~s}$ per channel);

3. Re-filling of the channels ( $5 \mathrm{~s}$ per channel, repeated 3 times).

After the first filling of the ten microfluidic channels and between each re-filling step, the array of permanent magnets was actuated for $30 \mathrm{~s}$. By following this fluidic/magnetic procedure, the sample was incubated in each channel for a total time of $5 \mathrm{~min}$. At the end of the assay procedure, the channels were washed with a procedure consisting of $15 \mathrm{~s}$ of buffer flowing per channel repeated 5 times. The total assay time comprising binding and washing was $16 \mathrm{~min}$.

For the regeneration step, after filling the fluidic line from the solution vial to the switch with the regeneration solution (SDS $0.25 \%, \mathrm{pH} 2.5$ ), a volume of $10 \mu \mathrm{L}$ of this solution was sequentially injected in each microfluidic channel with a sequence of short pulses assuring that its permanence in each channel was $60 \mathrm{~s}$. The washing step after the regeneration was conducted as reported before for the washing after the binding phase.

The fluorescence emitted by the sensing layer immobilised within the microfluidic channels was recorded every
$2 \mathrm{~s}$ as the average of 8 acquired values, using a photodiode integration time of $250 \mathrm{~ms}$.

\section{Results and discussion}

\section{Design and implementation of the optical device}

As illustrated in 'The optical device', the core of the optical device was the microfluidic chip with the optical components designed and optimised to achieve the necessary performances for its use in the analysis of clinical samples. The different components of the device together with their design and implementation are illustrated in the following sections.

\section{The microfluidic optical chip}

The 3D scheme of the microfluidic optical chip is shown in Fig. 3a. It comprised three parts (Fig. 3b): the Zeonor ${ }^{\circledR}$ bottom foil (dimensions $75.3 \mathrm{~mm} \times 30.4 \mathrm{~mm} \times 0.19 \mathrm{~mm}$, refractive index $n=1.53$ ), the injection-molded Zeonex ${ }^{\circledR}$ top part $(75.3 \mathrm{~mm} \times 25.4 \mathrm{~mm} \times 1.5 \mathrm{~mm}, n=1.51)$ and the double-sided adhesive tape $(75.3 \mathrm{~mm} \times 25.4 \mathrm{~mm} \times 0.14 \mathrm{~mm}$, $\mathrm{n}=1.49$ ), where the microchannels were manufactured by laser cutting. The flow channel depth, fixed by the adhesive tape thickness, is $140 \mu \mathrm{m}$, the width is $500 \mu \mathrm{m}$ and the length of the straight part is $14 \mathrm{~mm}$.

The upper part of the Zeonor ${ }^{\circledR}$ foil was functionalised with amino groups to allow the immobilisation of the MPA and CsA-O- $\mathrm{CO}_{2} \mathrm{H}$ derivatives (see 'Materials and protocols') so that, concerning the flow channel, the bioactive surface of the channel was only the bottom one. In addition, compared to the Zeonex ${ }^{\circledR}$ top part and the adhesive tape, the Zeonor ${ }^{\circledR}$ material of the bottom part of the chip had a higher refractive index.

By this means, the Zeonor ${ }^{\circledR}$ foil could be used as an optical waveguide element due to its higher refractive index: the excitation light, coming from an external source (see next subsection), could propagate inside the foil by total internal reflection (TIR) and could excite, by its evanescent field, the fluorescent sensing layer immobilised on the bottom of the microfluidic channels. A scheme of the cross section of the chip is depicted in Fig. 3c, where the optical path of the fluorescence rays is sketched in yellow. The Zeonex ${ }^{\circledR}$ part topping each microfluidic channel was shaped so that its side walls form an angle of $60^{\circ}$ with the horizontal line. As the fluorescence was not isotropic, due to the closeness of the fluorophore to the interface [32], the emission signal coming from the FMPs bound to the biosensing layer was mainly coupled inside the denser plastic foil with an angle close to the critical angle at the interface Zeonor ${ }^{\circledR}$-buffer solution $\left(\sim 61^{\circ}\right)$. Thus, after a reflection at the Zeonor ${ }^{\circledR}$-air 
Fig. 3 a 3D scheme of the microfluidic chip. b The three components of the optical chip. From left to right: double-sided adhesive tape with laser-cut microchannels, molded Zeonex ${ }^{\circledR}$ top part with integrated fluidic ports and Zeonor $^{\circledR}$ bottom foil. c Magnified view of the cross section of the microfluidic chip depicting two of the channels (the yellow lines represent the optical path of the fluorescence rays)
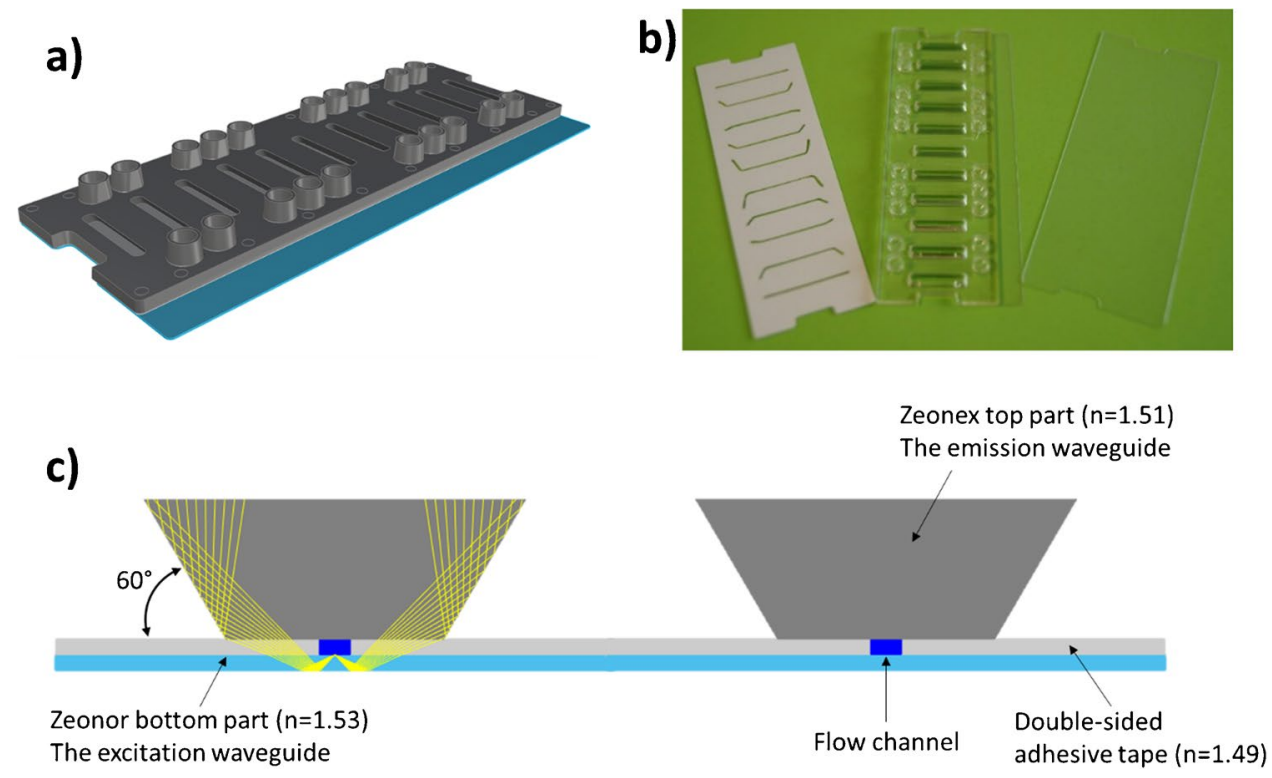

interface, the fluorescence signal mainly hit the top part of the chip in normal direction, thanks to the believed lateral sides. In this way, the fluorescence collection by the photodetectors located on top of the chip (see next subsection) was optimised.

\section{The optoelectronic excitation and detection module}

The excitation light is generated by a $30 \mathrm{~mW}$ TEM00 CW diode-pumped solid-state laser emitting at $589 \mathrm{~nm}$ (CNI MGL-III-589, Changchun New Industries Optoelectronics Technology) coupled to a bundle of eleven fibres (Molex Polymicro FVP150165195, high-OH silica/silica core diameter $150 \mu \mathrm{m}$, cladding diameter $165 \mu \mathrm{m}$, polyimide buffer $195 \mu \mathrm{m}, \mathrm{NA} 0.22$ ). The laser side of the bundle is terminated in circular shape and mounted on a micrometric alignment holder (Thorlabs K5X1 5-Axis Locking Kinematic Mount) facing the laser source. On the other end, ten fibres are directly faced to the chip by butt coupling them to the lateral side of the Zeonor ${ }^{\circledR}$ foil and aligned with the direction of the microfluidic channel (Fig. S1b and c). The eleventh fibre is interfaced to an external photodiode (Thorlabs SM05PD1B large area mounted silicon photodiode) for compensating possible laser power fluctuations. The rendering of the excitation module of the POCT device together with details of the fibre coupling and of the microfluidic chip is given in Fig. S1 of the supplementary information.

The detection module, which allowed to completely segregate the fluorescence generated in each of the ten microchannels, was formed by an array of ten optical filters (Schott RG645, coloured glass long pass filters) placed within a black anodised aluminium filter holder for straylight blocking and elimination of the cross-talk between adjacent filters, ten photodetectors (one for each microchannel) and the printed circuit board (PCB) equipped with spring-loaded connectors for the electrical connections with the photodetectors and with SMA RF connectors for the electronic boards. An exploded view of the detection module is provided in the supplementary information (Fig. S2a). The photodetectors were thin film nip-diodes made of hydrogenated amorphous silicon (a-Si:H) [33]. The main advantages of these a-Si:H-based thin film photodetectors were their low dark current and variable spectral response, the possibility to deposit detector elements at low temperature directly onto different superstrates (e.g. glass) or substrates (e.g. metal foil and silicon wafer), in particular with respect to microfluidic components, and their low mass production cost, which made them a good solution for the detection of very small radiation levels originating from low concentration fluorophore emission [34]. One of the ten photodiode series used in this system is depicted in Fig. S2b. The active area (36.3 $\mathrm{mm}^{2}$ ) and the two pads used for the electrical connections with the spring-loaded pins placed on the PCB board are visible in the pictured a-Si:H photodiode.

\section{Permanent magnet moving system}

A module with a permanent magnet array equipped with vertical motion was specifically developed for the device (Fig. S3a). The module was designed to be compatible with the integrated system and consists of a holder with 10 small neodymium-iron-boron permanent magnets (PM) (Supermagnete, Gottmadingen, Germany) with dimensions $10 \mathrm{~mm} \times 3$ $\mathrm{mm} \times 2 \mathrm{~mm}$ and a micro servo motor (5087MH HV, Hitec, USA), controlled by PC that allowed the vertical motion of the magnets. Here, the PM array was properly designed to 
have each magnet in correspondence with each microfluidic channel of the optical chip (Fig. S3b) and the typical actuation time of the motor was of the order of 0.1-0.5 s.

\section{The fluidic system}

Commercial pumps, valves and flow sensors (Fluigent ${ }^{\circledR}$ ) were used for the fabrication of the fluidics module. The technology provided by Fluigent ${ }^{\circledR}$ was based on a pressuredriven system that enabled the pulse-less flow actuation of fluids by pressure regulation.

The scheme of the fluidic system is depicted in the diagram of Fig. 4. The selection of each channel and the sequential filling of the ten channels of the microfluidic chip was controlled by two bidirectional 11-port/10-way valves (Fluigent ${ }^{\circledR}$ M-Switch ${ }^{\mathrm{TM}}$ ), indicated with V1 and V2 in Fig. 4. Two additional valves were used for the selection of the sample from the patient line or of the reagent/washing/perfusate lines (V3) and the selection of the sample/chemicals line or of the buffer line (V4). Two flow sensors (Fluigent ${ }^{\circledR}$ Flow Unit, $0-80 \mu \mathrm{L} / \mathrm{min}$ flow rate range), located in upstream of the mixer, accurately checked the flow rate. Additionally, the pressurisation of the waste vial could allow the forward and reverse flow inside the chip microchannels and inside the mixer. A suitable mixer, for mixing and incubating the sample with the antibody-coated FMPs outside the measuring chip, was specifically developed by microfluidic ChipShop and fabricated by injection molding in transparent thermoplastic polymer (Topas $\left.{ }^{\circledR}\right)$. Its scheme with inlet and outlet channels on both sides, two equal $40 \mu \mathrm{L}$ reaction chambers and a herringbone structure to facilitate mixing is provided in the supplementary information (Fig. S4).

\section{The software}

A commercial tablet PC (Motion ${ }^{\circledR}$ C5te, Motion Computing Inc) specifically designed for use in hospital environment was chosen as user interface. Communication with the POCT device was possible through a physical connection via either USB or ethernet ports. A 32-bit $\mathrm{ARM}^{\circledR} \mathrm{Cortex}^{\circledR}$-M0 $\mathrm{PSoC}^{\circledR} 4$ microcontroller was used for the electronic main boards while Python ${ }^{\circledR}$ was adopted for the firmware development along with the libraries Thinker, Serial and Matplotlib. For the control of fluidics components, the firmware was based on the Fluigent ${ }^{\circledR}$ MFCS ${ }^{\mathrm{TM}}$ Series SDK, which enabled access to low-level control of the components.

\section{The chip loading-engaging module}

The connection of the microfluidic optical chip with the microfluidic module was performed by means of a microfluidic manifold (dimensions: $83 \mathrm{~mm}$ in length, $36 \mathrm{~mm}$ in width and $13 \mathrm{~mm}$ in height) that engaged the 20 mini-Luer connections of the chip by exerting a suitable pressure (Fig. S5a). The manifold contained the optical filters and photodiodes and it was moved by a stepper motor linear actuator (L5918S2008-T10X2, Nanotec) that provided the necessary torque for the chip-manifold engaging (Fig. S5b). The chip loading was performed manually thanks to a sliding
Fig. 4 Schematic view of the fluidic system based on Fluigent ${ }^{\circledR}$ pressure-driven microfluidics pumps

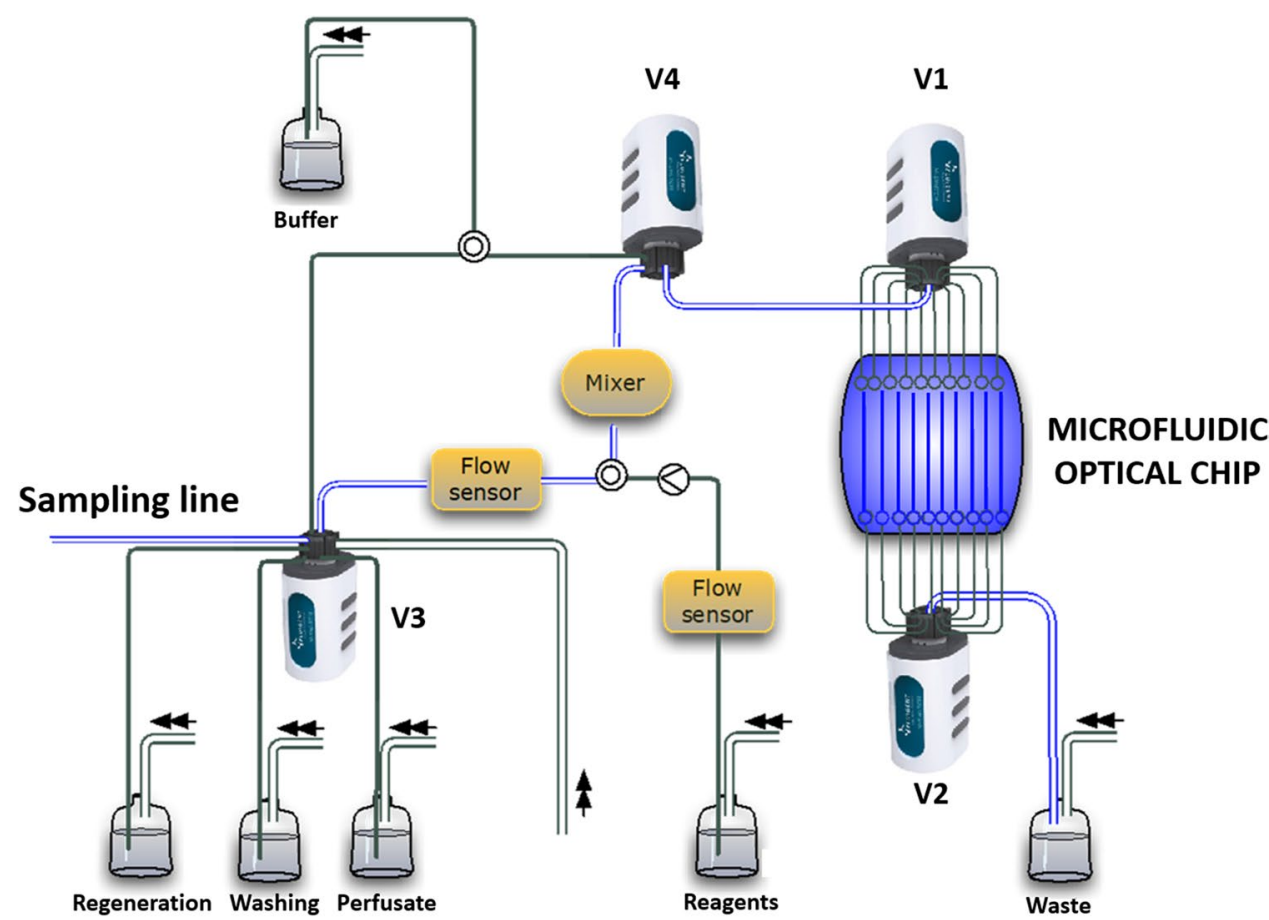


loading tray that allowed the firm positioning of the chip and the accurate alignment of the chip with the fluidic manifold and consequently with the photodiodes. The manifold also ensured the automatic alignment of the chip with the excitation fibres.

\section{Component optimisation and measurements with the integrated device}

The correct working of the FMPs regarding their interaction with the sensing layer and their capability to accelerate the assay was investigated by coupling the optical chip with the permanent magnet moving system shown in the supplementary information (Fig. S3a) and then evaluating the fluorescence associated with the microfluidic channels by acquiring an image of the complete channel with an inverted fluorescence microscope Zeiss AxioObserver.Z1 ( $5 \times$ objective, $\lambda_{\mathrm{ex}} 625 \mathrm{~nm}$, integration time $3 \mathrm{~s}$ ).

A suspension of anti-MPA antibody-coated FMPs was pumped into two microfluidic channels coated with tacrolimus or MPA, respectively, using a peristaltic pump at a flow rate of $4 \mu \mathrm{L} / \mathrm{min}$ according to the following protocol:

- Filling the channels with the FMPs suspension;

- Stopping the flow for 30 seconds;

- Raising the permanent magnet array until contacting the chip;

- Allowing interaction with the microchannel surface for an established time;

- Lowering of the magnet array and flowing off the FMPs for $30 \mathrm{~s}$ at $4 \mu \mathrm{L} / \mathrm{min}$.

These steps were repeated three times and then the channels were washed with PBST (PBS containing $0.05 \%$ of Tween 20) for $4 \mathrm{~min}$ at $200 \mu \mathrm{L} / \mathrm{min}$.

The images were analysed by using the microscope software and evaluating the densitometric value (average grey level of the image pixels) over the selected area corresponding to the whole channel. The average densitometric values were then evaluated by subtracting the background value corresponding to a blank and not-used channel.
Two different interaction times, $30 \mathrm{~min}$ and $5 \mathrm{~min}$, were used to verify the capability to perform the assay in shorter times and thus increase the frequency of measurements, which is an essential aspect in TDM. As shown in Table 1, in the absence of a magnetic trap the fluorescence intensity decreased with decreasing the interaction time, but the specific/non-specific ratio did not change. Thanks to the magnetic trapping achieved with the 10-magnet array, the fluorescence intensity from the channel increased two-fold for the same interaction time (Table 1) and, more interestingly, a higher specific/non-specific ratio was obtained, demonstrating the benefits derived from the use of the magnet array. The increased specific/non-specific ratio could be due to a combination of factors, including the decreased interaction time which fosters the specific binding with respect to the non-specific interaction, the different diffusion rates, and the affinity balance between the specific elements of the immunoassay.

Figure 5 shows the realised integrated optical device (dimensions: $28 \mathrm{~cm}$ in height, $36 \mathrm{~cm}$ in length and $45 \mathrm{~cm}$ in width $45 \mathrm{~cm}$; weight: $20 \mathrm{~kg}$ ). A thorough characterisation of its performance was carried out to ensure the reliability of the device before performing any assay.

The optical chip designed with the excitation provided by the evanescent wave propagating along the Zeonor foil allowed the geometric separation of the excitation and emission light paths, but any influence coming from the laserscattered excitation light had to be avoided in order to allow highly sensitive fluorescence measurements. Because of the small distance of the photodetectors from the chip and, therefore, from any potential scattering sources arriving from different directions, the scattered excitation stray light could not be filtered using interference filters exhibiting a strong angular dependency and thus long-pass absorption glass filters were preferable [35]. These filters were inexpensive and exhibited angle-independent spectral filtering properties. As shown in Fig. S2a in the supplementary information, a black filter holder was used to further shield the photodiodes from any other additional unwanted scattered light, and to avoid that the fluorescence generated in one microfluidic channel could reach the adjacent photodetectors, each of them
Table 1 Fluorescence intensity in arbitrary units, given as densiometric value, on two different microfluidic channels coated with tacrolimus and MPA, respectively, measured after the injection of anti-MPA antibody-coated magnetic particles for two different interaction times, with and without using the 10-magnet array

\begin{tabular}{llll}
\hline $\begin{array}{l}\text { Immunosuppressant immobilised on the chan- } \\
\text { nel surface }\end{array}$ & Interaction time 30 min/No magnet & Interaction time 5 min/No magnet & $\begin{array}{l}\text { Interaction time } \\
5 \text { min/10-magnet } \\
\text { array }\end{array}$ \\
\hline Tacrolimus (non-specific) & 19.3 & 5.4 & 11.7 \\
MPA (specific) & 39.1 & 10.7 & 68.2 \\
Specific/non- specific ratio & 2.0 & 2.0 & 5.8 \\
\hline
\end{tabular}


Fig. 5 Actual optical integrated device interfaced to the tablet PC

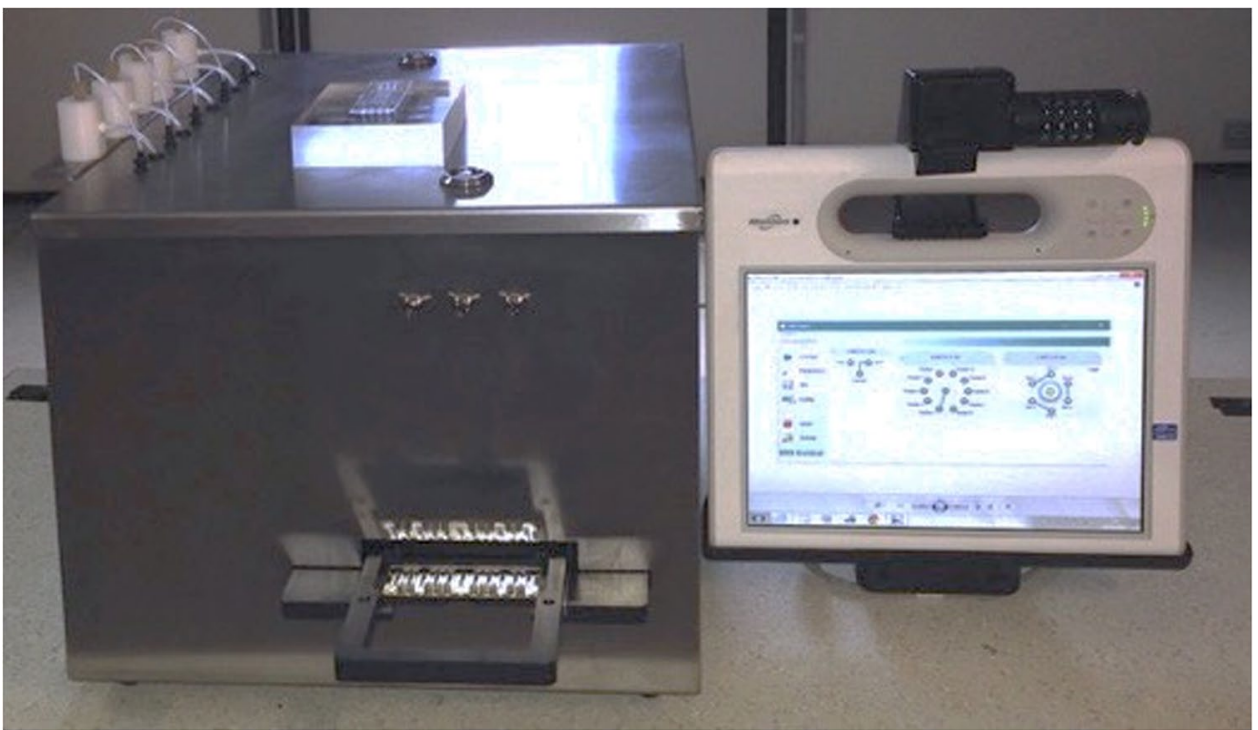

dedicated to the detection of the fluorescence coming from the adjacent channels.

The absence of any crosstalk between adjacent microfluidic channels was carefully verified. For this purpose, one channel (channel 7) of the chip was modified with MPA via the carbodiimide coupling method ('Materials and protocols') and the signals coming from channel 7 and from the adjacent channels (channels 6,8 and 9) were acquired after their filling with PBS (light grey bars in Fig. 6) and set to 1. Channel 7 was then filled with the FMPs modified with anti-MPA antibody for $30 \mathrm{~min}$. The signals acquired after the subsequent channel washing were normalized to the original signals when all channels were filled with only PBS (no fluorescence) (dark grey bars in Fig. 6). The relative variation of the signals in the four channels is shown in Fig. 6: the observed variations within the error bars in channels 6 , 8 and 9 and the strongly increased fluorescence signal of FMPs-loaded channel 7 excluded any fluorescence crosstalk between adjacent channels of the chip.

The optoelectronic and mechanical stability of the device was verified by measuring the signal coming from a chip with eight channels filled with buffer for $2 \mathrm{~h}$, without performing any fluidic procedure. In this way, the only measured contribution arised from the scattered excitation light, and any fluctuations in the detected signals had to be ascribed to fluctuations of the optoelectronics (source and/ or detector fluctuations) and/or to micrometre movements/ misalignments of the mechanical parts. As shown in Table 2,
Fig. 6 Evaluation of the crosstalk between the microfluidic/ optical detection channels (ch). Light gray bars: normalized fluorescence from all the channels filled with PBS; dark gray bars: fluorescence from the FMP attached to the bottom surface $\left(\right.$ Zeonor $\left.^{\circledR}\right)$ of $\operatorname{ch} 7$ microfluidic channel

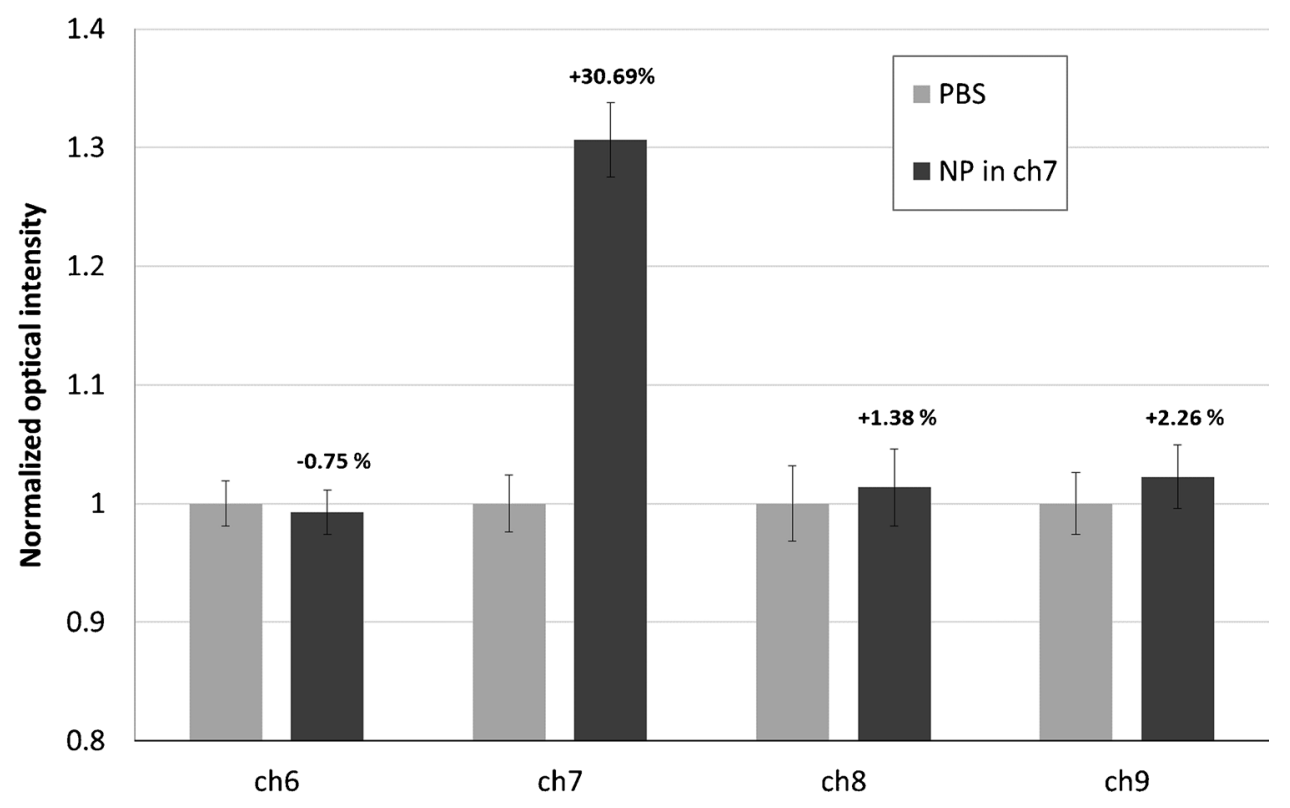


Table 2 Statistical analysis of the signals acquired in stable fluidic conditions on eight channels of the chip for $2 \mathrm{~h}$

\begin{tabular}{lllllllll}
\hline & ch2 & ch3 & ch4 & ch5 & ch6 & ch7 & ch8 & ch9 \\
\hline Mean value of the signal & 290350 & 345750 & 296940 & 185060 & 154340 & 150050 & 127990 & 152950 \\
Standard deviation & 6540 & 7040 & 6680 & 1970 & 7950 & 865 & 420 & 1850 \\
Relative error & $2.3 \%$ & $2.0 \%$ & $2.2 \%$ & $1.1 \%$ & $5.2 \%$ & $0.6 \%$ & $0.3 \%$ & $1.2 \%$ \\
\hline
\end{tabular}

a standard deviation less than $2.5 \%$ was observed for all the microchannels except for channel 6 (ch6), where the standard deviation was $5.2 \%$, confirming the good stability of the device.

The reliability of the chip loading-engaging procedure described above was verified by loading and removing the same chip five times and comparing the acquired signal coming from four microfluidic channels (channels 3-6) filled with PBS buffer. The measured signals summarised in Fig. 7, with their average and the standard deviation values, were characterised by very low fluctuations demonstrating the good repeatability of the adopted procedure. The absolute difference in optical signals between the different channels is probably due to a non-homogenous alignment of the eleven fibres bundle and the laser excitation beam and to possible different losses suffered by different fibres. The consequence is that the fibres do not carry exactly the same optical power, but the illumination of each channel is sufficiently stable in time, so that the signals of the ten channels can be normalized after a calibration.

Lipofundin samples spiked with CsA and MPA and real microdialysate samples, resulting from ex vivo microdialysis of whole blood samples from immunosuppressed patients, were tested with the developed optical device.

A calibration curve for CsA was obtained by using $20 \%$ Lipofundin spiked with different concentrations of CsA. The different solutions were tested sequentially with a regeneration step between each assay. Three assay procedures were conducted for each concentration; the blank sample contained only $20 \%$ Lipofundin and corresponded to the maximum signal with no binding inhibition, as tested at the beginning of the series and every 5 measurements with other concentrations. The different concentrations were tested with a random distribution in the series including the measurements of the real microdialysate samples.

A total of 21 regeneration cycles was conducted with a recovery signal ranging from 75 to $90 \%$ with respect to the previous background point. This recovery variation did not affect the reproducibility of the signal shift for each concentration and consequently the $\mathrm{B}_{\mathrm{x}} / \mathrm{B}_{0}$ ratio indicating the binding inhibition (ratio among the signal obtained with a particular concentration of the antigen and that without the antigen).

The calibration curve for CsA, obtained with this series of measurements, is shown in Fig. 8a. With the parameters of the best logistic fitting curve $\left(A_{1}=0.999, A_{2}=0.146, x_{0}=1.02\right.$, $p=1.75$, where $A_{1}$ and $A_{2}$ are the asymptotes of the fit, $x_{0}$ is the value of the CsA concentration for which the fluorescence signal is equal to the $50 \%$ of the dynamic range and $p$ gives the slope of the curve for $x=x_{0}$; adjusted $R$-squared $=0.9998$ ) and considering the error on the zero concentration a limit of detection (LOD; calculated from the logistic fitting by using three times the error on the blank) of 0.48 $\mathrm{ng} / \mathrm{mL}$ was obtained [36]. The working range, evaluated by considering the concentration range between 10 and $90 \%$ of the difference between the upper and lower asymptote, the so-called dynamic range [37], resulted in $0.57-10 \mathrm{ng} /$ $\mathrm{mL}$. To evaluate the reproducibility, the coefficient of variation $(\mathrm{CV} \%)$ of each tested concentration was calculated by considering the standard deviation resulting from the different measurements conducted on each concentration: by
Fig. 7 Detected optical signals from four different channels filled with PBS buffer after successively loading the chip inside the optical device five times. The standard deviations $(\sigma)$ of the five different acquired signals are indicated

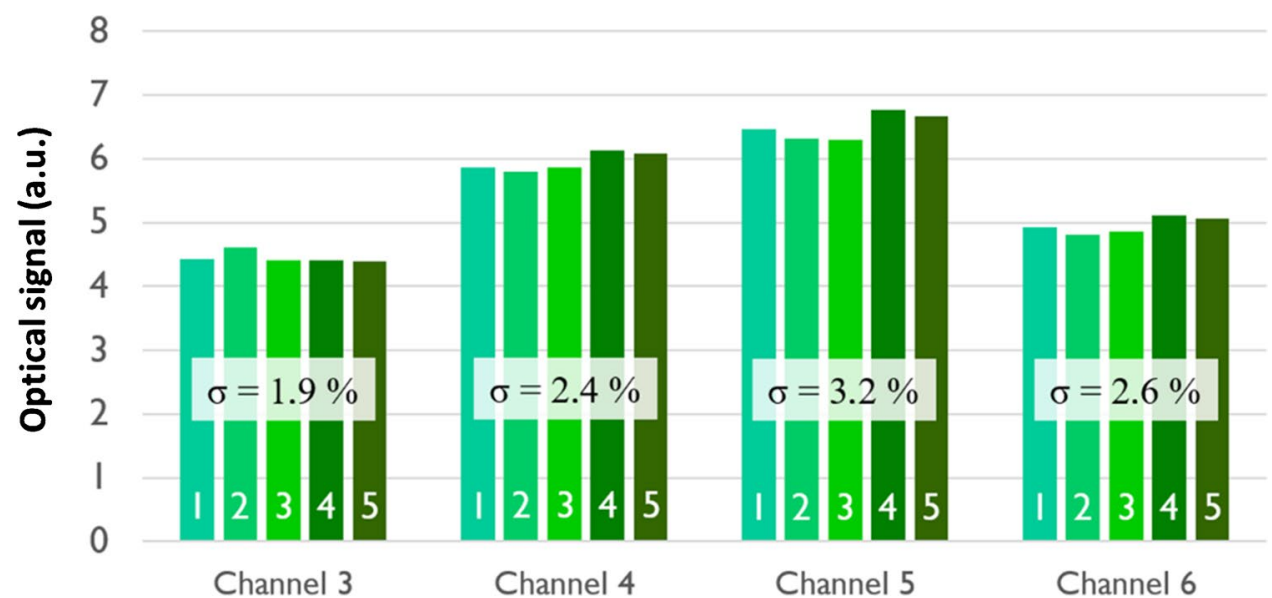




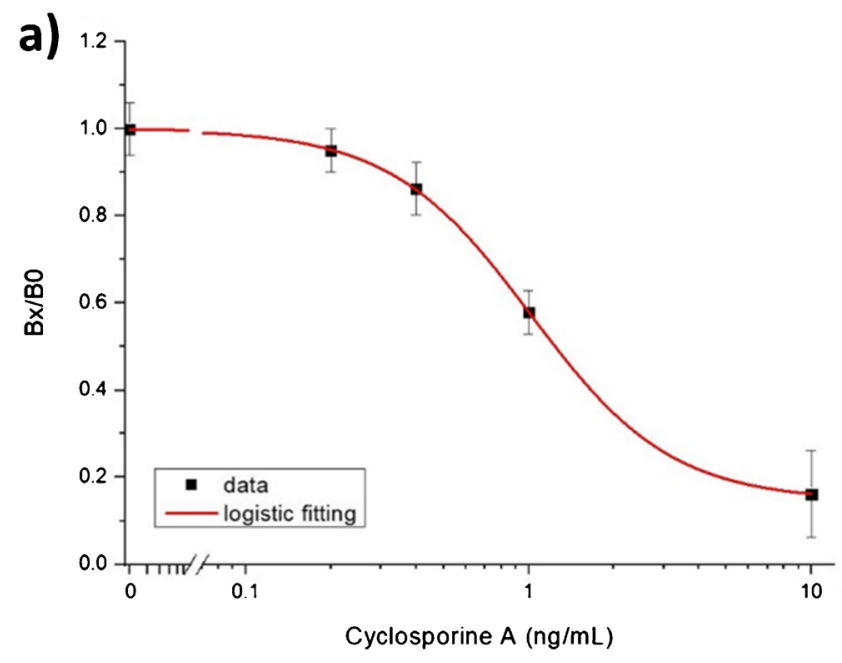

Fig. 8 a Calibration curve for CsA obtained with the optical device using FMPs coated with anti-CsA antibody and CsA diluted in $20 \%$ Lipofundin. b Calibration curve for MPA obtained with the opti-

averaging the resulting $\mathrm{CV} \%$, a value of $7 \%$ was obtained which demonstrates a good reproducibility of the assay.

Two real microdialysate samples from patients were also tested and their CsA concentrations were calculated from the calibration fitting curve of Fig. 8a. Concentrations of $0.50 \pm 0.03 \mathrm{ng} / \mathrm{mL}$ and $0.57 \pm 0.04 \mathrm{ng} / \mathrm{mL}$ were obtained for the two samples, which were coherent with the expected concentration of free CsA in the microdialysate samples, according to the total CsA-to-CsA in microdialysate ratio reported in ref [25].

The same procedure was adopted to obtain the calibration curve for MPA (Fig. 8b). In this case, the signals from the four MPA-modified channels were considered.

With the parameters of the logistic fitting curve ( $A_{1}=0.993, A_{2}=0.076, x_{0}=1.96, p=1.87$; adjusted $R$-squared $=0.9918$ ) and considering the error on the blank, a LOD of $0.79 \mathrm{ng} / \mathrm{mL}$ was calculated with an average $\mathrm{CV}$ of $7 \%$ and a working range of $0.87-17 \mathrm{ng} / \mathrm{mL}$. The concentration of MPA detected in the two real microdialysate samples $(3.7 \mathrm{ng} / \mathrm{mL}$ and $30 \mathrm{ng} / \mathrm{mL}$, respectively) showed a higher variability than that of CsA. Due to the better dialysability of MPA and the broader concentration range of MPA [25], these significantly higher concentrations were reasonable.

\section{Conclusions}

A fluorescence-based POCT device was developed for the simultaneous automatic measurement of a mixture of immunosuppressants towards its application in TDM. This capability was achieved, thanks to the development of a 10-channel microfluidic chip, designed to optimize the collection of the laser-induced fluorescence from a bioaffinity layer

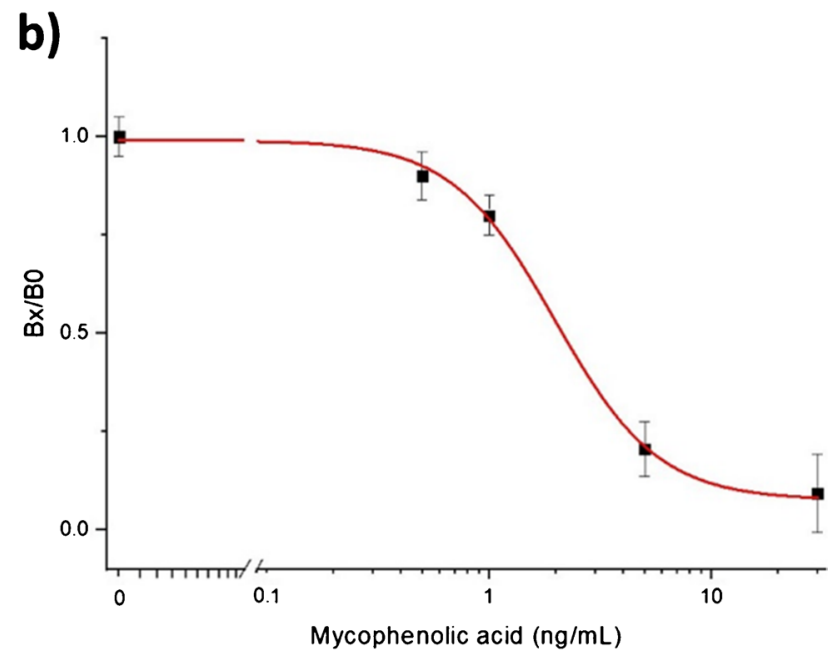

cal device by using FMPs coated with anti-MPA antibody and MPA diluted in $20 \%$ Lipofundin

after interacting with the immunosuppressant of interest in the presence of tailored fluoromagnetic particles. The high efficiency of the signal collection allowed to cover the working range of the immunosuppressants requested for clinical applications with promising results for CsA and MPA while the use of FMPs led to a significant reduction of the interaction time of the analyte with the biosensing layer, yielding an overall assay time of just $16 \mathrm{~min}$. There is still some work to be done, as the achieved working ranges do not meet completely the requirements of the clinicians: even if sufficiently sensitive to the low levels of drug concentrations in microdialysates, the ranges have to be extended to higher values. This is particularly true for MPA which is highly dialyzable due to its high polarity and low molecular weight and for which values as high as many tens of $\mathrm{ng} / \mathrm{mL}$ have been found in kidney transplanted patients [25]. The microfluidic circuit integrated in the device allowed the automatic sample handling, from the mixing with the necessary chemicals to the interaction with the biosensing layer. All these features make the developed unit a potential POCT device for immunosuppressant monitoring transplanted patients and for TDM in general, representing an important step forward in the field since the traditional approach of sample withdrawal followed by an analysis in an external laboratory is unable to meet the required short turn-around-time (TAT). Strategies based on sparse blood sampling within longer time windows have been developed for clinical purposes and have been shown to substantially improve the patient's outcome. Regular pharmacokinetic monitoring requires repetitive blood sampling and our device can offer an essential support to solve this crucial problem with respect to the determination of the correct dosage of the administered drugs. It should also be pointed out that a connection between the novel device 
microfluidics with the microfluidic line coming out from an intravascular microdialysis catheter could be readily implemented making the quasi-continuous TDM in transplanted patients more than just a dream.

Supplementary Information The online version contains supplementary material available at https://doi.org/10.1007/s00216-021-03847-x.

Funding The authors acknowledge the European Community for its funding within the framework of the project Nanodem - Nanophotonic device for multiple therapeutic drug monitoring (GA 318372) of the 7th Framework Programme.

\section{Declarations}

Ethics approval and consent to participate Research involving human subjects was conducted according to the Declaration of Helsinki, after approval by the Ethics Committee of the Klinikum rechts der Isar of the Technische Universität München (46/14). All patients gave written informed consent.

Competing interests The author GG is an editor of the journal, but as such was not involved in the peer review of this article. The other authors declare no conflict of interest.

Open Access This article is licensed under a Creative Commons Attribution 4.0 International License, which permits use, sharing, adaptation, distribution and reproduction in any medium or format, as long as you give appropriate credit to the original author(s) and the source, provide a link to the Creative Commons licence, and indicate if changes were made. The images or other third party material in this article are included in the article's Creative Commons licence, unless indicated otherwise in a credit line to the material. If material is not included in the article's Creative Commons licence and your intended use is not permitted by statutory regulation or exceeds the permitted use, you will need to obtain permission directly from the copyright holder. To view a copy of this licence, visit http://creativecommons.org/licenses/by/4.0/.

\section{References}

1. Luppa PB, Müller C, Schlichtiger A, Schlebusch H. Point-ofcare testing (POCT): current techniques and future perspectives. TRAC-Trend Anal Chem. 2011. https://doi.org/10.1016/j.trac. 2011.01.019.

2. Luppa PB, Bietenbeck A, Beaudoin C, Giannetti A. Clinically relevant analytical techniques, organizational concepts for application and future perspectives of point-of-care testing. Biotechnol Adv. 2016. https://doi.org/10.1016/j.biotechadv.2016.01.003.

3. Thaler M, Luppa PB. Highly sensitive immunodiagnostics at the point of care employing alternative recognition elements and smartphones: hype, trend, or revolution? Anal Bioanal Chem. 2019. https://doi.org/10.1007/s00216-019-01974-0.

4. Cremers S, Guha N, Shine B. Therapeutic drug monitoring in the era of precision medicine: opportunities! Br J Clin Pharmacol. 2016. https://doi.org/10.1111/bcp.13047.

5. Ates HC, Roberts JA, Lipman J, AEG C, Urban GA, Dincer C. On-site therapeutic drug monitoring. Trends biotechnol. 2020. https://doi.org/10.1016/j.tibtech.2020.03.001.
6. Pearce CM, Resmini M. Towards point of care systems for the therapeutic drug monitoring of imatinib. Anal Bioanal Chem. 2020. https://doi.org/10.1007/s00216-020-02545-4.

7. Ponticelli C, Glassock RJ. Prevention of complications from use of conventional immunosuppressants: a critical review. J Nephrol. 2019. https://doi.org/10.1007/s40620-019-00602-5.

8. Freudenberger K, Hilbig U, Gauglitz G. Recent advances in therapeutic drug monitoring of immunosuppressive drugs. TRACTrend Anal Chem. 2016. https://doi.org/10.1016/j.trac.2015.11. 016.

9. Udomkarnjananun S, Francke MI, De Winter BCM, Mulder MB, Baan CC, Metselaar HJ, den Hoed CM, Hesselink DA. Therapeutic drug monitoring of immunosuppressive drugs in hepatology and gastroenterology. Best Pract Res Cl Ga. 2021. https://doi.org/ 10.1016/j.bpg.2021.101756.

10. Zhang Y, Zhang R. Recent advances in analytical methods for the therapeutic drug monitoring of immunosuppressive drugs. Drug Test Anal. 2018. https://doi.org/10.1002/dta.2290.

11. Martínez-Chávez A, Rosing H, Hillebrand M, Tibben M, Schinkel AH, Beijnen JH. Development and validation of a bioanalytical method for the quantification of the CDK4/6 inhibitors abemaciclib, palbociclib, and ribociclib in human and mouse matrices using liquid chromatography-tandem mass spectrometry. Anal Bioanal Chem. 2019. https://doi.org/10.1007/ s00216-019-01932-w.

12. Saint-Marcoux F, Woillard J-B, Jurado C, Marquet P. Lessons from routine dose adjustment of tacrolimus in renal transplant patients based on global exposure. Ther Drug Monit. 2013. https:// doi.org/10.1097/FTD.0b013e318285e779.

13. Marquet P, Åsberg A. Individualizing transplant therapy. In: Jelliffe RW, Nelly M, editors. Individualized drug therapy for patients. Boston: Academic Press; 2017. https://doi.org/10.1016/ B978-0-12-803348-7.00016-2.

14. Radzevičienė A, Marquet $P$, Maslauskienė $R$, Vaičiūnienė R, Kaduševičius E, Stankevičius E. Analyses of AUC $(0-12)$ and C0 compliances within therapeutic ranges in kidney recipients receiving cyclosporine or tacrolimus. J Clin Med. 2020. https://doi.org/ 10.3390/jcm9123903.

15. Taddeo A, Prim D, Bojescu E-D, Segura J-M, Pfeifer ME. Pointof-care therapeutic drug monitoring for precision dosing of immunosuppressive drugs. J Appl Lab Med. 2020. https://doi.org/10. 1093/jalm/jfaa067.

16. Etienne I, Toupance O, Bénichou J, Thierry A, Al Najjar A, de Ligny BH, Le Meur Y, Westeel P-F, Marquet P, François A, Hellot M-F, Godin M. A 50\% reduction in cyclosporine exposure in stable renal transplant recipients: renal function benefits. Nephrol Dial Transplant. 2010. https://doi.org/10.1093/ndt/gfq135.

17. Naesens M, Lerut E, Damme BV, Vanrenterghem Y, Kuypers DRJ. Tacrolimus exposure and evolution of renal allograft histology in the first year after transplantation. Am J Transplant. 2007. https://doi.org/10.1111/j.1600-6143.2007.01892.x.

18. Woillard JB, de Winter BC, Kamar N, Marquet P, Rostaing L, Rousseau A. Population pharmacokinetic model and Bayesian estimator for two tacrolimus formulations--twice daily Prograf and once daily Advagraf. Br J Clin Pharmaco. 2011. https://doi. org/10.1111/j.1365-2125.2010.03837.x.

19. Le Meur Y, Büchler M, Thierry A, Caillard S, Villemain F, Lavaud S, Etienne I, Westeel P-F, De Ligny BH, Rostaing L, Thervet E, Szelag JC, Rérolle J-P, Rousseau A, Touchard G, Marquet P. Individualized mycophenolate mofetil dosing based on drug exposure significantly improves patient outcomes after renal transplantation. Am J Transplant. 2007. https://doi.org/10. 1111/j.1600-6143.2007.01983.x.

20. Daher Abdi Z, Prémaud A, Essig M, Alain S, Munteanu E, Garnier F, Le Meur Y, Marquet P, Rousseau A. Exposure to mycophenolic acid better predicts immunosuppressive efficacy than exposure to 
calcineurin inhibitors in renal transplant patients. Clin Pharmacol Ther. 2014. https://doi.org/10.1038/clpt.2014.140.

21. Miyamoto T, Takashima S, Kato K, Takase K, Yoshimoto G, Yoshida S, Henzan H, Osaki K, Kamimura T, Iwasaki H, Eto T, Teshima T, Nagafuji K, Akashi K. Comparison of cyclosporine and tacrolimus combined with mycophenolate mofetil in prophylaxis for graft-versus-host disease after reduced-intensity umbilical cord blood transplantation. Int J Hematol. 2017. https://doi. org/10.1007/s12185-016-2093-0.

22. Sandmaier BM, Kornblit B, Storer BE, Olesen G, Maris MB, Langston AA, Gutman JA, Petersen SL, Chauncey TR, Bethge WA, Pulsipher MA, Woolfrey AE, Mielcarek M, Martin PJ, Appelbaum FR, Flowers MED, Maloney DG, Storb R. Addition of sirolimus to standard cyclosporine plus mycophenolate mofetilbased graft-versus-host disease prophylaxis for patients after unrelated non-myeloablative haemopoietic stem cell transplantation: a multicentre, randomised, phase 3 trial. Lancet Haematol. 2019. https://doi.org/10.1016/S2352-3026(19)30088-2.

23. Dasgupta A. Usefulness of monitoring free (unbound), concentrations of therapeutic drugs in patient management. Clin Chim Acta. 2007. https://doi.org/10.1016/j.cca.2006.08.026.

24. Bittersohl H, Herbinger J, Wen M, Renders L, Steimer W, Luppa PB. Simultaneous determination of protein-unbound cyclosporine A and mycophenolic acid in kidney transplant patients using liquid chromatography-tandem mass spectrometry. Ther Drug Monit. 2017. https://doi.org/10.1097/FTD.0000000000000392.

25. Weber S, Tombelli S, Giannetti A, Trono C, O'Connell M, Wen M, Descalzo AB, Bittersohl H, Bietenbeck A, Marquet P, Renders L, Orellana G, Baldini F, Luppa PB. Immunosuppressant quantification in intravenous microdialysate - towards novel quasi-continuous therapeutic drug monitoring in transplanted patients. Clin Chem Lab Med. 2021. https://doi.org/10.1515/cclm-2020-1542.

26. Osaka T, Matsunaga T, Nakanishi T, Arakaki A, Niwa D, Iida H. Synthesis of magnetic nanoparticles and their application to bioassays. Anal Bioanal Chem. 2006. https://doi.org/10.1007/ s00216-005-0255-7.

27. Wu K, Su D, Saha R, Liu J, Chugh VK, Wang J-P. Magnetic particle spectroscopy: a short review of applications using magnetic nanoparticles. ACS Appl Nano Mater. 2020. https://doi.org/10. 1021/acsanm.0c00890.

28. Duan N, Wu S, Zhu C, Ma X, Wang Z, Yu Y, Jiang Y. Dual-color upconversion fluorescence and aptamer-functionalized magnetic nanoparticles-based bioassay for the simultaneous detection of Salmonella Typhimurium and Staphylococcus aureus. Anal Chim Acta. 2012. https://doi.org/10.1016/j.aca.2012.02.011.

29. Garzón V, Pinacho DG, Bustos R-H, Garzón G, Bustamante S. Optical biosensors for therapeutic drug monitoring. Biosensors. 2019. https://doi.org/10.3390/bios9040132.

30. Poschenrieder A, Thaler M, Junker R, Luppa PB. Recent advances in immunodiagnostics based on biosensor technologies-from central laboratory to the point of care. Anal Bioanal Chem. 2019. https://doi.org/10.1007/s00216-019-01915-x.

31. Salis F, Descalzo AB, Benito-Peña E, Moreno-Bondi MC, Orellana G. Highly fluorescent magnetic nanobeads with a remarkable stokes shift as labels for enhanced detection in immunoassays. Small. 2018. https://doi.org/10.1002/smll.201703810.

32. Baldini F, Carloni A, Giannetti A, Porro G, Trono C. An optical PMMA biochip based on fluorescence anisotropy: application to C-reactive protein assay. Sensor Actuat B-Chem. 2009. https:// doi.org/10.1016/j.snb.2008.08.027.

33. Berner M, Rothemund R, Vollmer S, Schubert M, Hilbig U, Gauglitz G. Silicon thin film photo-detectors for multi-channel fluorescence detection in a microfluidic point-of-care testing device. Phys Status Solidi A. 2016. https://doi.org/10.1002/pssa.201532953.

34. Berner M, Hilbig U, Schubert MB, Gauglitz G. Laser-induced fluorescence detection platform for point-of-care testing. Meas Sci Technol. 2017. https://doi.org/10.1088/1361-6501/aa7810.

35. Berneschi S, Trono C, Bernini R, Giannetti A, Persichetti G, Testa G, Tombelli S, Baldini F. A waveguide absorption filter for fluorescence measurements. Sensor Actuat B-Chem. 2019. https://doi. org/10.1016/j.snb.2018.10.011.

36. Gauglitz G. Analytical evaluation of sensor measurements. Anal Bioanal Chem. 2018. https://doi.org/10.1007/s00216-017-0624-z.

37. Albrecht C, Kaeppel N, Gauglitz G. Two immunoassay formats for fully automated CRP detection in human serum. Anal Bioanal Chem. 2008. https://doi.org/10.1007/s00216-008-2093-x.

Publisher's note Springer Nature remains neutral with regard to jurisdictional claims in published maps and institutional affiliations. 